\title{
Qualidade de Vida e Reabilitação dos Pacientes Oncológicos
}

\author{
Quality of Life and Rehabilitation in Oncologic Patients
}

\section{RESUMO}

O texto discute o projeto de extensão da Escola de Enfermagem de Ribeirão Preto Adriana Cristina da Universidade de São Paulo, ligado ao Grupo de Pesquisa Estudo e Reabilitação do paciente cirúrgico e oncológico, cadastrado no $\mathrm{CNPq}$, que tem o objetivo de atender os pacientes com câncer em tratamento quimioterápico proporcionando terapias complementares como relaxamento com imagem guiada e acupuntura para diminuir os sintomas da doença e do tratamento. Trata-se de estudo quase experimental, com grupo-controle e grupo de intervenção, desenvolvido na unidade de quimioterapia do Nicolussi, Camila Picharillo, Daiane Arruda Saraiva, JULiana Maria de Paula, Liyoko Okino e Namie Orino SaWAda Hospital Beneficência Portuguesa de Ribeirão Preto. Os resultados demonstram que as terapias complementares diminuem os sintomas, melhorando a qualidade de vida dos pacientes.

Palavras-chave: Qualidade de vida. Qualidade de vida relacionada à saúde. Terapia complementar.

\section{ABSTRACT}

This is the community service project of the University of São Paulo at Ribeirão Preto College of Nursing linked to the Research Group "Study and Rehabilitation of the surgical and cancer patient, enrolled in the National Council for Scientific and Technological Development (CNPq), that aims to assist patients with cancer undergoing chemotherapy providing complementary therapies such as relaxation with guided imagery and acupuncture to lessen the symptoms of the disease and treatment. Quasi-experimental study with intervention and control group developed in the chemotherapy unit at the Hospital Beneficência Portuguesa of Ribeirão Preto. The results demonstrate that the complementary therapies lessen the symptoms improving patients' quality of life. 
Keywords: Quality of life. Health-related quality of life. Complementary therapy.

\section{INTRODUÇÃO}

O projeto de extensão "Qualidade de vida e reabilitação dos pacientes oncológicos" está vinculado ao "Grupo de Estudo e Reabilitação do paciente cirúrgico e oncológico", cadastrado no CNPq desde 1990. Ele tem como finalidade o atendimento de pacientes com câncer em tratamento quimioterápico com intervenções de terapia complementar para diminuir os sinais e sintomas da doença e do tratamento, melhorando, consequentemente, a qualidade de vida relacionada à saúde desses pacientes. Além da interface com a pesquisa, essa atividade de extensão também visa ao envolvimento de alunos de graduação e pós-graduação, complementando a formação e o aprimoramento profissional. Esse projeto foi iniciado em 2005 e originou várias dissertações de mestrado e artigos científicos como: PAULA, J. M.; SONOBE, H. M.; NICOLUSSI, A. C.; ZAGO, M. M. F.; SAWADA, N. O. Sintomas de depressão nos pacientes com câncer de cabeça e pescoço em tratamento radioterápico: um estudo prospectivo. Rev. Latino Americana de Enfermagem, 20(2)mar/abril, 2012; NICOLUSSI, A. C.; SAWADA, N. O. Qualidade de vida de pacientes com câncer de mama em terapia adjuvante. Revista Gaúcha de Enfermagem, v. 4, pp. 759-766, 2011; SAWADA, N. O.; ZAGO, M. M. F.; GALVÃO, C. M.; CARDOZO, F. M. C.; ZANDONAI, A. P.; OKINO, L.; NICOLUSSI, A. C. The outcomes of visualization and acupuncture on the quality of life of adult cancer patients receiving chemotherapy. Cancer $\operatorname{Nursing}^{T M}$ v. 33, n. 5, 2010; ZANDONAI, A. P.; CARDOZO, F. M. C.; NIETO, I. N. G.; SAWADA, N. O. Qualidade de vida nos pacientes oncológicos: revisão integrativa da literatura latino-americana. Revista Eletrônica de Enfermagem 12(3), pp. 554-561, 2010; NICOLUSSI, A. C.; SAWADA, N. O. Qualidade de vida de pacientes com câncer colorretal em terapia adjuvante. Acta Paulista de Enfermagem 22(2), pp. 155-161, 2009. Essas pesquisas demonstraram a importância da investigação da qualidade de vida relacionada à saúde de pacientes oncológicos para o planejamento do processo de reabilitação e os resultados da terapia complementar no alívio de sintomas nos pacientes em quimioterapia.

Nas últimas décadas, o câncer tem sido considerado um problema de saúde pública mundial, uma vez que tem aumentado a sua prevalência dentro das doenças crônicas não transmissíveis, requerendo grandes investimentos financeiros e representando ônus institucional e social para o país. Portanto, faz-se necessário o controle epidemiológico dos casos e programas de prevenção e reabilitação. A reabilitação do paciente com câncer é considerada uma das áreas de maior relevância para as ciências da saúde, entre elas a enfermagem. $\mathrm{O}$ tratamento cirúrgico do câncer e os outros procedimentos terapêuticos (radioterapia e quimioterapia) têm resultado na diminuição da mortalidade e da morbidade; entretanto, existe a preocupação com o status funcional e a qualidade de vida dessas pessoas. Assim, a reabilitação do paciente com câncer é um processo contínuo, com a finalidade de maximizar as capacidades dos indivíduos dentro das limitações impostas pela doença e pelo tratamento.

Qualidade de vida relacionada à Saúde (QVRS) é uma experiência subjetiva, 
multidimensional, que envolve um resumo da avaliação de atributos positivos e negativos, tais como condições de saúde e doença, que caracteriza uma vida [5].

O termo QV é comumente usado em intercambio com QVRS, bem como o termo resultado relatado pelo paciente (PROs) na literatura especializada. Os PROs são medidas de resultados relatados pelo paciente que incluem QV e QVRS, mas também podem incluir outros itens, tais como sintomas físicos e psicológicos. Entretanto, QVRS pode ser definida como QV dentro do contexto de saúde e doença. Dessa forma, esses dois termos podem ser intercambiados na área do cuidado oncológico [1].

Os estudos sobre qualidade de vida relacionada à saúde têm auxiliado na compreensão da natureza e extensão de problemas funcionais e psicossociais durante a trajetória da doença. Outro ponto importante é a avaliação da terapêutica e das intervenções que proporcionam uma melhor assistência a esses pacientes e, consequentemente, uma reabilitação precoce.

As terapias complementares também têm sido desenvolvidas, paralelamente ao tratamento convencional, com resultados bastante significativos na melhora da qualidade de vida desses pacientes. Rhodes e McDaniel [7] levantam os vários tipos de intervenções não farmacológicas que podem ser usadas em combinação ou como adjuvante da terapia medicamentosa. Dentre elas, ressaltam o controle da dieta e ambiente, acupuntura, musicoterapia, relaxamento e visualização.

O objetivo dessa pesquisa foi avaliar a terapia complementar (acupuntura e relaxamento com visualização guiada), sobre os sintomas de ansiedade e depressão nos pacientes oncológicos sobre tratamento quimioterápico e o impacto sobre a sua qualidade de vida.

\section{MATERIAL E MÉTODO}

\section{Tipo de Estudo}

Trata-se de um estudo com abordagem quantitativa, de delineamento quase experimental, de corte longitudinal e prospectivo.

\section{Local do Estudo}

O estudo foi realizado no Centro Especializado de Oncologia (CEON) do Hospital Sociedade Portuguesa de Beneficência.

\section{Coleta de Dados}

Para a coleta de dados foi utilizado um questionário de identificação, construído para a coleta dos dados sociodemográficos (como sexo, idade, estado civil, procedência, profissão/ocupação, nível de escolaridade e religião) e dos dados clínicos e terapêuticos (como diagnóstico, realização e tipo de cirurgia, realização de radioterapia, protocolo, sessões e efeitos colaterais da quimioterapia). Para a avaliação da QV, utilizamos o instrumento European Organization for Research and Treatment of Cancer (EORTC) Quality of Life Questionnaire-Core3o (QLQ-C30), que é um questionário de QVRS 
devidamente validado para a população brasileira por Brabo, em 2006, para uso específico em pacientes com câncer. O Inventário de Depressão de Beck foi utilizado para avaliar a Ansiedade e Depressão, adaptado no Brasil por Gorestein e Andrade [2].

\section{Análise dos Dados}

Para a análise dos dados, utilizamos o software Statistical Package for Social Science versão 17.0 (SPSS for Windows). Utilizamos o teste de consistência interna Alpha de Cronbach para testar a confiabilidade dos instrumentos. Fizemos também o cáculo da média e desvio padrão para análise descritiva dos dados. O Teste t de Student foi utilizado para calcular os vários momentos de aplicação dos instrumentos no grupo de intervenção. A Prova de Kruskal-Wallis, enfim, foi usada para comparar os grupos controle e de intervenção quanto às variáveis $\mathrm{QV}$, ansiedade e depressão.

\section{RESULTADOS}

Realizamos, entre fevereiro de 2009 e junho de 2011, cerca de 300 atendimentos com base na intervenção com as terapias complementares (relaxamento com imagem guiada e acupuntura) em um total de 32 pacientes (GI), além de termos realizado a aplicação dos instrumentos em 24 pacientes no grupo-controle.

$\mathrm{Na}$ amostra inicial dos grupos de intervenção e controle, tivemos o total de 56 pacientes, sendo a maioria do sexo feminino em ambos os grupos; na faixa etária entre 40 e 60 anos no GI e entre 60 e 80 no GC; casados; aposentados ou donas de casa; procedentes de Ribeirão Preto; que completaram o ensino Fundamental; e de religião católica em ambos os grupos, como demonstrado na Tabela 1.

Na Tabela 2, mostramos a caracterização clínica e terapêutica dos grupos e encontramos os diversos tipos de câncer: de mama, gástrico, intestinal, do aparelho reprodutor feminino (útero, ovário), do aparelho reprodutor masculino (próstata), além dos cânceres de pulmão, de cabeça e pescoço, neurológicos (como glioblastoma e meduloblastoma), leucemias e linfomas, e cânceres de pele, inclusive melanomas, sendo que $25 \%$ dos pacientes tinham câncer metastático em ambos os grupos.

Quanto aos tratamentos realizados, observamos que a maioria dos pacientes realizou cirurgia e quimioterapia, e alguns realizaram radioterapia. Em um pequeno número de pacientes não foi possível identificar o protocolo de quimioterapia, ou já haviam terminado este tratamento, mas estavam em seguimento e, por isso, foram mantidos no estudo. Vários protocolos de quimioterapia foram identificados e também estão listados na Tabela 2. 
Tabela 1 Características sóciodemográficas da amostra, CEON - Ribeirão Preto, 2009-2011.

\begin{tabular}{|c|c|c|c|c|}
\hline & \multirow{2}{*}{ CARACTERÍSTICAS } & \multicolumn{2}{|l|}{ GI } & \multirow{2}{*}{$\begin{array}{c}\text { AMOSTRA } \\
\text { TOTAL } \\
\mathrm{N}(\%)\end{array}$} \\
\hline & & N (\%) & N (\%) & \\
\hline \multirow[t]{3}{*}{ SEXO } & Feminino & $20(62,5)$ & $14(58,3)$ & $34(60,7)$ \\
\hline & Masculino & $12(37,5)$ & $10(41,7)$ & $22(39,3)$ \\
\hline & TOTAL & $22(100)$ & $24(100)$ & $56(100)$ \\
\hline \multirow[t]{5}{*}{ FAIXA ETÁRIA } & 20 ê3/4 40 anos & $1(3,1)$ & $0(0,0)$ & $1(1,8)$ \\
\hline & 40 ê3/4 60 anos & $20(62,5)$ & $11(45,8)$ & $31(55,3)$ \\
\hline & 60 ê3/4 80 anos & $11(34,4)$ & $12(50,0)$ & $23(41,1)$ \\
\hline & $\geq 80$ & $0(0,0)$ & $1(4,2)$ & $1(1,8)$ \\
\hline & TOTAL & $32(100)$ & $24(100)$ & $56(100)$ \\
\hline \multirow[t]{5}{*}{ ESTADO CIVIL } & Solteiro & $5(15,6)$ & $3(12,5)$ & $8(14,3)$ \\
\hline & Casado & $21(65,5)$ & $15(62,5)$ & $36(64,3)$ \\
\hline & Viúvo & $2(6,3)$ & $5(20,8)$ & $7(12,5)$ \\
\hline & Divorciado/amasiado & $4(12,5)$ & $1(4,2)$ & $5(8,9)$ \\
\hline & TOTAL & $32(100)$ & $24(100)$ & $56(100)$ \\
\hline \multirow[t]{12}{*}{ PROFISSÃO } & Aposentados & $7(21,9)$ & $6(25,0)$ & $13(23,2)$ \\
\hline & Dona de casa & $5(15,6)$ & $9(37,5)$ & $14(25,0)$ \\
\hline & $\begin{array}{l}\text { Diarista, manicure, } \\
\text { cabeleireira }\end{array}$ & $3(9,4)$ & $1(4,2)$ & $4(7,2)$ \\
\hline & Comerciantes & $4(12,5)$ & $4(16,7)$ & $8(14,3)$ \\
\hline & Agricultores & $1(3,1)$ & $0(0,0)$ & $1(1,8)$ \\
\hline & $\begin{array}{l}\text { Motorista, pedreiro, } \\
\text { eletricista }\end{array}$ & $5(15,6)$ & $3(12,5)$ & $8(14,3)$ \\
\hline & $\begin{array}{l}\text { Engenheiro, arquiteto, } \\
\text { publicitário }\end{array}$ & $2(6,3)$ & $1(4,2)$ & $3(5,3)$ \\
\hline & Professor & $1(3,1)$ & $0(0,0)$ & $1(1,8)$ \\
\hline & $\begin{array}{l}\text { Serviço técnico e/ou } \\
\text { auxiliar }\end{array}$ & $1(3,1)$ & $0(0,0)$ & $1(1,8)$ \\
\hline & $\begin{array}{l}\text { Atendente } \\
\text { administrativo }\end{array}$ & $1(3,1)$ & $0(0,0)$ & $1(1,8)$ \\
\hline & $\begin{array}{l}\text { Profissionais da saúde } \\
\text { universitários }\end{array}$ & $2(6,3)$ & $0(0,0)$ & $2(3,5)$ \\
\hline & TOTAL & $32(100)$ & $24(100)$ & $56(100)$ \\
\hline
\end{tabular}




\begin{tabular}{|c|c|c|c|c|}
\hline & \multirow[t]{2}{*}{ CARACTERÍSTICAS } & GI & GC & $\begin{array}{c}\text { AMOSTRA } \\
\text { TOTAL }\end{array}$ \\
\hline & & N (\%) & N (\%) & N (\%) \\
\hline \multirow[t]{4}{*}{ CIDADE } & Ribeirão Preto & $28(87,5)$ & $13(54,2)$ & $41(73,2)$ \\
\hline & $\begin{array}{l}\text { Região de Ribeirão } \\
\text { Preto }\end{array}$ & $4(12,5)$ & $10(41,7)$ & $14(25,0)$ \\
\hline & Outras regiões & $0(0,0)$ & $1(4,2)$ & $1(1,8)$ \\
\hline & TOTAL & $32(100)$ & $24(100)$ & $56(100)$ \\
\hline \multirow{4}{*}{$\begin{array}{l}\text { NÍVEL DE } \\
\text { ESCOLARIDADE }\end{array}$} & Ensino Fundamental & $17(53,1)$ & $17(70,8)$ & $34(60,7)$ \\
\hline & Ensino Médio & $8(25,0)$ & $5(20,8)$ & $13(23,2)$ \\
\hline & Ensino Superior & $7(21,9)$ & $2(8,3)$ & $9(16,1)$ \\
\hline & TOTAL & $32(100)$ & $24(100)$ & $56(100)$ \\
\hline \multirow[t]{5}{*}{ RELIGIÃO } & Ateu & $1(3,1)$ & $2(8,3)$ & $3(5,3)$ \\
\hline & Católico & $23(71,9)$ & $17(70,8)$ & $40(71,4)$ \\
\hline & Espírita & $2(6,3)$ & $0(0,0)$ & $2(3,6)$ \\
\hline & Evangélico/cristão & $6(18,8)$ & $5(20,8)$ & $11(19,7)$ \\
\hline & TOTAL & $32(100)$ & $24(100)$ & $56(100)$ \\
\hline
\end{tabular}

Tabela 2 Características clínico-terapêuticas da amostra, CEON - Ribeirão Preto, 2009-2011.

\begin{tabular}{|c|c|c|c|c|}
\hline & \multirow[t]{2}{*}{ CARACTERÍSTICAS } & \multicolumn{2}{|l|}{ GI } & \multirow{2}{*}{$\begin{array}{c}\begin{array}{c}\text { AMOSTRA } \\
\text { TOTAL }\end{array} \\
\text { N (\%) }\end{array}$} \\
\hline & & N (\%) & N (\%) & \\
\hline \multirow{11}{*}{$\begin{array}{l}\text { DIAGNÓSTICO } \\
\text { (LOCALIZAÇÃO } \\
\text { DO CÂNCER) }\end{array}$} & Mama & $11(34,4)$ & $2(8,3)$ & $13(23,2)$ \\
\hline & Gástrico & $3(9,4)$ & $6(25,0)$ & $9(16,1)$ \\
\hline & Intestinal & $3(9,4)$ & $9(37,5)$ & $12(21,4)$ \\
\hline & Reprodutor feminino & $2(6,3)$ & $1(4,2)$ & $3(5,3)$ \\
\hline & Pulmão/mediastino & $3(9,4)$ & $0(0,0)$ & $3(5,3)$ \\
\hline & $\begin{array}{l}\text { Reprodutor/genital } \\
\text { masculino }\end{array}$ & $1(3,1)$ & $0(0,0)$ & $1(1,8)$ \\
\hline & Cabeça e pescoço & $4(12,5)$ & $2(8,3)$ & $6(10,7)$ \\
\hline & Neurológico & $0(0,0)$ & $1(4,2)$ & $1(1,8)$ \\
\hline & Leucemias/linfomas & $3(9,4)$ & $0(0,0)$ & $3(5,3)$ \\
\hline & Pele e melanomas & $2(6,3)$ & $3(12,5)$ & $5(9,0)$ \\
\hline & TOTAL & $32(100)$ & $24(100)$ & $56(100)$ \\
\hline
\end{tabular}




\begin{tabular}{|c|c|c|c|c|}
\hline & \multirow{2}{*}{ CARACTERÍSTICAS } & \multicolumn{2}{|l|}{ GI } & \multirow{2}{*}{$\begin{array}{c}\text { AMOSTRA } \\
\text { TOTAL } \\
\mathrm{N}(\%)\end{array}$} \\
\hline & & $N(\%)$ & N (\%) & \\
\hline \multirow[t]{3}{*}{ METÁSTASE } & $\operatorname{Sim}$ & $8(25,0)$ & $6(25,0)$ & $14(25,0)$ \\
\hline & Não & $24(75,0)$ & $18(75,0)$ & $42(75,0)$ \\
\hline & TOTAL & $32(100)$ & $24(100)$ & $56(100)$ \\
\hline \multirow{3}{*}{$\begin{array}{l}\text { REALIZAÇÃO DE } \\
\text { CIRURGIA }\end{array}$} & $\operatorname{Sim}$ & $25(78,1)$ & $19(79,2)$ & $44(78,6)$ \\
\hline & Não & $7(21,9)$ & $5(20,8)$ & $12(21,4)$ \\
\hline & TOTAL & $32(100)$ & $24(100)$ & $56(100)$ \\
\hline \multirow[t]{8}{*}{$\begin{array}{l}\text { TIPO DE } \\
\text { CIRURGIA }\end{array}$} & $\begin{array}{l}\text { Não realizaram/não } \\
\text { soube informar }\end{array}$ & $7(21,9)$ & $6(25,0)$ & $13(23,2)$ \\
\hline & Biópsia & $6(18,8)$ & $1(4,2)$ & $7(12,5)$ \\
\hline & $\begin{array}{l}\text { Retirada de tumor/ } \\
\text { nódulos e/ou } \\
\text { adjacências }\end{array}$ & $4(12,5)$ & $2(8,3)$ & $6(10,7)$ \\
\hline & $\begin{array}{l}\text { Retirada parcial do } \\
\text { órgão afetado e/ou } \\
\text { adjacências }\end{array}$ & $8(25,0)$ & $12(50,0)$ & $20(35,7)$ \\
\hline & $\begin{array}{l}\text { Retirada total do } \\
\text { órgão afetado e/ou } \\
\text { adjacências }\end{array}$ & $5(15,6)$ & $1(4,2)$ & $6(10,7)$ \\
\hline & Cirurgia paliativa & $1(3,1)$ & $2(8,3)$ & $3(5,3)$ \\
\hline & Transplante & $1(3,1)$ & $0(0,0)$ & $1(1,8)$ \\
\hline & TOTAL & $32(100)$ & $24(100)$ & $56(100)$ \\
\hline \multirow{3}{*}{$\begin{array}{l}\text { REALIZAÇÃO DE } \\
\text { RADIOTERAPIA }\end{array}$} & Sim & $11(34,4)$ & $7(29,2)$ & $18(32,1)$ \\
\hline & Não & $21(65,6)$ & $17(70,8)$ & $38(67,9)$ \\
\hline & TOTAL & $32(100)$ & $24(100)$ & $56(100)$ \\
\hline \multirow{3}{*}{$\begin{array}{l}\text { REALIZAÇÃO DE } \\
\text { QUIMIOTERAPIA }\end{array}$} & Sim & $24(75,0)$ & $22(91,7)$ & $46(82,1)$ \\
\hline & $\begin{array}{l}\text { Não faz/não } \\
\text { identificado }\end{array}$ & $8(25,0)$ & $2(8,3)$ & $10(17,9)$ \\
\hline & TOTAL & $32(100)$ & $24(100)$ & $56(100)$ \\
\hline
\end{tabular}




\begin{tabular}{|c|c|c|c|c|}
\hline & \multirow[t]{2}{*}{ CARACTERÍSTICAS } & GI & GC & $\begin{array}{l}\text { AMOSTRA } \\
\text { TOTAL }\end{array}$ \\
\hline & & N (\%) & N (\%) & N (\%) \\
\hline \multirow{25}{*}{$\begin{array}{l}\text { PROTOCOLO DE } \\
\text { QUIMIOTERAPIA }\end{array}$} & Não identificado & $8(25,0)$ & $2(8,3)$ & $10(17,9)$ \\
\hline & $\begin{array}{l}\text { Paclitaxel + } \\
\text { carboplatina }\end{array}$ & $0(0,0)$ & $1(4,2)$ & $1(1,8)$ \\
\hline & Paclitaxel + cisplatina & $2(6,3)$ & $0(0,0)$ & $2(3,5)$ \\
\hline & Cisplatina & $4(12,5)$ & $1(4,2)$ & $5(9,0)$ \\
\hline & $\begin{array}{l}\text { Carboplatina } \\
+ \text { docetaxel/ + } \\
\text { gencitabina + } \\
\text { cisplatina }\end{array}$ & $1(3,1)$ & $0(0,0)$ & $1(1,8)$ \\
\hline & $\begin{array}{l}\text { Cisplatina + } \\
\text { fluorouracil (5FU) }\end{array}$ & $1(3,1)$ & $1(4,2)$ & $2(3,5)$ \\
\hline & Leucovorin $+5 F U$ & $3(9,4)$ & $11(45,8)$ & $14(25,0)$ \\
\hline & Paclitaxel & $2(6,3)$ & $0(0,0)$ & $2(3,5)$ \\
\hline & Doxorrubicina & $1(3,1)$ & $0(0,0)$ & $1(1,8)$ \\
\hline & $\begin{array}{l}\text { Cisplatina }+ \\
\text { dacarbazina }+ \\
\text { carmustina }\end{array}$ & $1(3,1)$ & $0(0,0)$ & $1(1,8)$ \\
\hline & Gencitabina & $0(0,0)$ & $1(4,2)$ & $1(1,8)$ \\
\hline & $\begin{array}{l}\text { Cisplatina + 5FU + } \\
\text { paclitaxel }\end{array}$ & $1(3,1)$ & $1(4,2)$ & $2(3,5)$ \\
\hline & 5FU + doxorrubicina & $1(3,1)$ & $0(0,0)$ & $1(1,8)$ \\
\hline & Mitomicina $+5 F U$ & $0(0,0)$ & $1(4,2)$ & $1(1,8)$ \\
\hline & $\begin{array}{l}\text { Leucovorin }+5 F U+ \\
\text { oxaliplatina }\end{array}$ & $1(3,1)$ & $1(4,2)$ & $2(3,5)$ \\
\hline & alkeran (melfalano) & $1(3,1)$ & $0(0,0)$ & $1(1,8)$ \\
\hline & Dacarbazina (DTIC) & $0(0,0)$ & $1(4,2)$ & $1(1,8)$ \\
\hline & $\begin{array}{l}\text { ciclofosfamida }+ \\
\text { paclitaxel }\end{array}$ & $0(0,0)$ & $1(4,2)$ & $1(1,8)$ \\
\hline & faslodex (fulvestranto) & $1(3,1)$ & $0(0,0)$ & $1(1,8)$ \\
\hline & $\begin{array}{l}\text { adriamicina }+ \\
\text { bleomicina }+ \text { velbon }+ \\
\text { dacarbazina }\end{array}$ & $1(3,1)$ & $0(0,0)$ & $1(1,8)$ \\
\hline & Talidominda & $1(3,1)$ & $0(0,0)$ & $1(1,8)$ \\
\hline & $\begin{array}{l}\text { cisplatina + vinorelbine } \\
\text { (navelbine) }\end{array}$ & $1(3,1)$ & $0(0,0)$ & $1(1,8)$ \\
\hline & tamoxifeno & $1(3,1)$ & $0(0,0)$ & $1(1,8)$ \\
\hline & $5 F U$ & $0(0,0)$ & $2(8,3)$ & $2(3,5)$ \\
\hline & TOTAL & $32(100)$ & $24(100)$ & $56(100)$ \\
\hline
\end{tabular}




\begin{tabular}{|c|c|c|c|c|}
\hline & \multirow[t]{2}{*}{ CARACTERÍSTICAS } & GI & GC & $\begin{array}{c}\text { AMOSTRA } \\
\text { TOTAL }\end{array}$ \\
\hline & & N (\%) & N (\%) & N (\%) \\
\hline \multirow{7}{*}{$\begin{array}{l}\text { EFEITOS } \\
\text { COLATERAIS DA } \\
\text { QUIMIOTERAPIA }\end{array}$} & Assintomático & $18(56,3)$ & $21(87,5)$ & $39(69,6)$ \\
\hline & $\begin{array}{l}\text { Efeitos } \\
\text { gastrointestinais }\end{array}$ & $1(3,1)$ & $1(4,2)$ & $2(3,6)$ \\
\hline & Efeitos físicos & $2(6,3)$ & $2(8,3)$ & $4(7,1)$ \\
\hline & $\begin{array}{l}\text { Efeitos } \\
\text { gastrointestinais + } \\
\text { físicos }\end{array}$ & $9(28,1)$ & $0(0,0)$ & $9(16,1)$ \\
\hline & $\begin{array}{l}\text { Efeitos } \\
\text { gastrointestinais }+ \\
\text { emocionais }\end{array}$ & $1(3,1)$ & $0(0,0)$ & $1(1,8)$ \\
\hline & $\begin{array}{l}\text { Efeitos } \\
\text { gastrointestinais + } \\
\text { físicos + emocionais }\end{array}$ & $1(3,1)$ & $0(0,0)$ & $1(1,8)$ \\
\hline & TOTAL & $32(100)$ & $24(100)$ & $56(100)$ \\
\hline
\end{tabular}

Em relação à coleta dos instrumentos de qualidade de vida, 31 pacientes do GI e 23 pacientes no GC responderam, inicialmente, o instrumento QLQ-C30 (baseline). Devido a perdas, por motivos diversos, somente doze pacientes no GI e quatro, no GC, responderam-no ao final do estudo (seis meses), cujos resultados estão apresentados nas Tabelas 3 e 4.

Tabela 3 Média e desvio padrão das escalas do instrumento QLQ-C30 (baseline), Grupo de Intervenção e Grupo-Controle, CEON - Ribeirão Preto, 2009-2011.

\begin{tabular}{lll}
\hline \multirow{2}{*}{ ESCALAS E SINTOMAS } & GI (N=31) & GC (N=23) \\
\cline { 2 - 3 } & MÉDIA (DP) & MÉDIA (DP) \\
\hline Estado Geral de Saúde (EGS/OV) & $56,95(25,92)$ & $82,58(1,50)$ \\
\hline Função física (FF) & $62,64(25,12)$ & $70,08(30,31)$ \\
\hline Desempenho de papel (DP) & $51,74(37,09)$ & $66,08(37,38)$ \\
\hline Função emocional (FE) & $46,00(33,93)$ & $60,95(26,47)$ \\
\hline Função cognitiva (FC) & $55,03(38,99)$ & $84,30(25,29)$ \\
\hline Função social (FS) & $70,03(37,59)$ & $77,08(29,95)$ \\
\hline Fadiga (FAD) & $46,91(33,65)$ & $29,92(35,17)$ \\
\hline Náuseas e vômitos (NAV) & $25,24(28,49)$ & $5,06(12,73)$ \\
\hline Dor (Dor) & $56,43(37,43)$ & $26,79(32,46)$ \\
\hline Dispnéia (DIS) & $25,64(35,04)$ & $7,21(22,34)$ \\
\hline
\end{tabular}




\begin{tabular}{lcc}
\hline \multirow{2}{*}{ ESCALAS E SINTOMAS } & GI (N=31) & GC (N=23) \\
\cline { 2 - 3 } Insônia (INS) & MÉDIA (DP) & MÉDIA (DP) \\
\hline Perda de apetite (PAP) & $43,83(36,84)$ & $30,21(34,52)$ \\
\hline Constipação (CON) & $40,77(46,08)$ & $15,82(28,04)$ \\
\hline Diarréia (DIA) & $22,48(35,81)$ & $10,04(20,95)$ \\
\hline Dificuldades financeiras (DIF) & $12,80(23,73)$ & $5,78(21,65)$ \\
\hline
\end{tabular}

Tabela 4 Média e desvio padrão das escalas do instrumento QLQ-C30 (após seis meses), Grupo de Intervenção e Grupo-Controle, CEON - Ribeirão Preto, 2009-2011.

\begin{tabular}{lcc}
\hline \multirow{2}{*}{ ESCALAS E SINTOMAS } & GI (N=12) & GC (N=4) \\
\cline { 2 - 3 } Estado Geral de Saúde (EGS/OV) & MÉDIA (DP) & MÉDIA (DP) \\
\hline Função física (FF) & $64,55(18,17)$ & $77,07(20,83)$ \\
\hline Desempenho de papel (DP) & $68,66(22,47)$ & $80,25(19,70)$ \\
\hline Função emocional (FE) & $60,00(35,70)$ & $96,00(8,00)$ \\
\hline Função cognitiva (FC) & $60,95(29,12)$ & $52,25(16,93)$ \\
\hline Função social (FS) & $69,83(28,14)$ & $92,00(9,23)$ \\
\hline Fadiga (FAD) & $68,33(32,98)$ & $83,75(13,47)$ \\
\hline Náuseas e vômitos (NAV) & $32,37(30,10)$ & $16,65(21,25)$ \\
\hline Dor (Dor) & $13,87(18,56)$ & $12,50(25,00)$ \\
\hline Dispnéia (DIS) & $36,07(25,45)$ & $20,80(20,98)$ \\
\hline Insônia (INS) & $13,83(29,97)$ & $8,25(16,50)$ \\
\hline Perda de apetite (PAP) & $27,58(36,96)$ & $0,00(0,00)$ \\
\hline Constipação (CON) & $13,83(33,11)$ & $25,00(50,00)$ \\
\hline Diarréia (DIA) & $16,50(26,32)$ & $16,50(33,00)$ \\
\hline Dificuldades financeiras (DIF) & $8,25(20,51)$ & $8,25(16,50)$ \\
\hline
\end{tabular}

Notamos que os resultados melhoraram em várias escalas e sintomas para o GI, o que pode ser relacionado à prática das terapias complementares de relaxamento com imagem guiada e acupuntura, mesmo com a perda de mais de 50\% da amostra. Também notamos a melhora no GC. No entanto, como apenas quatro pacientes (17\%) responderam o questionário neste outro momento, não temos como analisar estes resultados, pois podem ser os mesmos pacientes que já consideravam ter uma boa qualidade de vida no início. 
Isso porque, normalmente, os pacientes que desistiam do estudo em ambos os grupos eram aqueles que já não estavam tão bem e confiantes com os tratamentos realizados.

Quanto ao Inventário de Depressão de Beck, conseguimos manter a amostra no início e final da coleta de dados com 32 respondentes no GI e 14 no GC e demonstramos os resultados nas Tabelas 5 e 6. Observamos que a maioria dos pacientes do GI e grande parte dos pacientes do GC estavam sem "depressão” no início, de acordo com os escores do instrumento e esta percentagem melhorou no GI, demonstrando que as terapias complementares foram efetivas, enquanto que no GC, os resultados ficaram estáveis.

Tabela 5 Frequência e percentagem do Inventário de Depressão de Beck (baseline), Grupos de Intervenção e Controle, CEON - Ribeirão Preto, 2009-2011.

\begin{tabular}{lcc}
\hline & GI & GC \\
\cline { 2 - 3 } ESCORES & $\mathbf{N}(\%)$ & $\mathbf{N}(\%)$ \\
\hline Sem Depressão & $18(56,3)$ & $11(45,8)$ \\
\hline Disforia & $6(18,85)$ & $2(8,3)$ \\
\hline Depressão & $8(25,0)$ & $1(4,2)$ \\
\hline TOTAL & $32(100,0)$ & $14(58,3)$ \\
\hline PERDA & $0(0,0)$ & $10(41,7)$ \\
\hline TOTAL & $32(100,0)$ & $24(100,0)$ \\
\hline
\end{tabular}

Tabela 6 Frequência e percentagem do Inventário de Depressão de Beck (após seis meses), Grupos de Intervenção e Controle, CEON - Ribeirão Preto, 2009-2011.

\begin{tabular}{lcc}
\hline \multirow{2}{*}{ ESCORES } & GI & GC \\
\cline { 2 - 3 } & $\mathbf{N}(\%)$ & $\mathbf{N}(\%)$ \\
\hline Sem Depressão & $20(62,5)$ & $11(45,8)$ \\
\hline Disforia & $4(12,5)$ & $2(8,3)$ \\
\hline Depressão & $8(25,0)$ & $1(4,2)$ \\
\hline TOTAL & $\mathbf{3 2}(100,0)$ & $\mathbf{1 4}(58,3)$ \\
\hline PERDA & $\mathbf{0 ( 0 , 0 )}$ & $\mathbf{1 0}(\mathbf{4 1 , 7 )}$ \\
\hline TOTAL & $\mathbf{3 2}(\mathbf{1 0 0 , 0 )}$ & $\mathbf{2 4}(\mathbf{1 0 0 , 0 )}$ \\
\hline
\end{tabular}

Continuamos a realização da coleta de dados pelas dificuldades encontradas com relação à permanência dos pacientes no tratamento complementar, o que prejudicou a análise dos dados a longo prazo, além de uma reforma na unidade de oncologia ter causado dificuldade no recrutamento de novos pacientes. Mesmo diante dessas dificuldades 
acreditamos que os resultados são promissores e que o relaxamento com imagem guiada e a acupuntura constituem-se em terapias complementares importantes no tratamento do paciente com câncer em tratamento quimioterápico. Cabe aos profissionais da saúde educarem e conscientizarem os pacientes sobre essas terapias, uma vez que esse tratamento é de baixo custo, sem efeitos colaterais e com resultados positivos sobre a qualidade de vida dos mesmos; além disso, as atividades de extensão fazem parte do tripé da universidade, associadas ao ensino e pesquisa, onde é possível transferir para a sociedade o conhecimento produzido e melhorar as condições de vida da população.

\section{REFERÊNCIAS BIBLIOGRÁFICAS}

[1] FAYERS, P. M.; MACHIN, D. Quality of life - the assessment, analysis and interpretation of patient-reported outcomes. 2. ed. Chichester: John Wiley \& Sons, 2007.

[2] GOREINSTEIN, C; ANDRADE, L. Validation of Portuguese version of Beck Depression Inventory and State-trait Anxiety Inventory in Brazilian subjects. Brazilian Journal of Medical and Biological Research, n. 29, pp. 453-457, 1996.

[3] NICOLUSSI, A. C.; SAWADA, N.O. Qualidade de vida de pacientes com câncer de mama em terapia adjuvante. Revista Gaúcha de Enfermagem, v. 4, pp. 759-766, 2011.

[4] _. Qualidade de vida de pacientes com câncer colorretal em terapia adjuvante. Acta Paulista de Enfermagem 22(2), pp. 155-161, 2009.

[5] PADILLA, G. V. et al. Defining the content domain of quality of life for cancer patients with pain. Cancer Nursing, v. 13, pp. 108-115, 1990.

[6] PAULA, J. M.; SONOBE, H. M.; NICOLUSSI, A. C.; ZAGO, M. M. F; SAWADA, N. O. Sintomas de depressão nos pacientes com câncer de cabeça e pescoço em tratamento radioterápico: um estudo prospectivo. Rev. Latino-Americana de Enfermagem, 20(2) mar/abril, 2012.

[7] RHODES, V.A; MCDANIEL, R. W. Nausea, vomiting, and retching: complex problems in palliative care. Cancer J. Clin., 51, pp. 232-248, 2001.

[8] SAWADA, N. O.; ZAGO, M. M. F.; GALVÃO, C. M.; CARDOZO, F. M. C.; ZANDONAI, A. P.; OKINO, L.; NICOLUSSI, A.C. The outcomes of visualization and acupuncture on the quality of life of adult cancer patients receiving chemotherapy. Cancer Nursing ${ }^{T M}$ v. 33, n. 5, 2010.

[9] ZANDONAI, A. P.; CARDOZO, F. M. C.; NIETO, I. N. G.; SAWADA, N. O. Qualidade de vida nos pacientes oncológicos: revisão integrativa da literatura latino-americana. Revista Eletrônica de Enfermagem 12(3), pp. 554-561, 2010.

ADRIANA CRISTINA NICOLUSSI aluna de doutorado do Programa Enfermagem Fundamental da Escola de Enfermagem de Ribeirão Preto da Universidade de São Paulo.

CAMILA PICHARILLO bolsista do Programa Aprender com Cultura e Extensão da Escola de Enfermagem de Ribeirão Preto da Universidade de São Paulo. 
DAIANE ARRUdA SARAIVA bolsista do Programa Aprender com Cultura e Extensão da Escola de Enfermagem de Ribeirão Preto da Universidade de São Paulo.

JULIANA MARIA DE PAULA aluna de iniciação científica CNPq da Escola de Enfermagem de Ribeirão Preto da Universidade de São Paulo.

LIYOKO OKINO médica Especialista em Acupuntura.

NAMIE OKINO SAWADA professora associada do Departamento de Enfermagem Geral e Especializada da Escola de Enfermagem de Ribeirão Preto da Universidade de São Paulo - Av. Bandeirantes, 3900 - Monte Alegre - CEP 14040-902 - Ribeirão Preto-SP - e-mail: sawada@eerp.usp.br. 